\title{
A Genetic Search in Frequency Space for Stabilizing Atoms by High-Intensity Laser Fields
}

\author{
Danny Barash, Ann E. Orel and V. Rao Vemuri \\ Department of Applied Science, University of California, Davis and Lawrence Livermore National Laboratories, Livermore, \\ California
}

The goal of this paper is to explore the power of stochastic search methods, in particular genetic algorithms, to solve a challenging problem in experimental physics. The problem is to find an optimum frequency to stabilize atoms by high-intensity laser fields. The standard approach to search for optimal laser parameters has been by trial and error. This is the first known application of a genetic algorithm technique to model atomic stabilization. Genetic algorithms worked well for this problem as a way to automate the search in a time efficient manner. A parallel platform is used to perform the genetic search efficiently. Locating the best frequency to achieve a suppression of ionization, which is predicted to occur at high intensities, can help design a laboratory experiment and tune to that frequency in order to identify a stabilization effect. The genetic algorithms did successfully identify this optimum frequency. It is indeed possible to extend the number of unknown tunable laser parameters, beyond searching merely over frequency space. For instance, optimal pulse shape and pulse duration can also be included. While conducting such a search in multi-dimensional parameter space, parallel genetic algorithms can offer an advantage to the tedious trial and error procedures.

\section{Introduction}

This paper aims at finding the optimum frequency via genetic algorithms (GA), for which an atomic stabilization effect is maximum. It is possible to search frequency space to locate this optimum based on the following fitness function:

$$
\begin{array}{r}
f(\omega)=\left(p 0_{I_{i+1}}-p 0_{I_{i}}\right)+\left(p 0_{I_{i+2}}-p 0_{I_{i}}\right)+\ldots \\
\quad+\left(p 0_{I_{i-1}}-p 0_{I_{i}}\right)+\left(p 0_{I_{i-2}}-p 0_{I_{i}}\right)+\ldots(1)
\end{array}
$$

The quantities in this expression, resulting from numerically solving the time-dependent Schrödinger equation (TDSE) for a laser-atom interaction, will briefly be explained at an introductory level. The focus of this paper, however, is on how to perform the genetic search given the above fitness function which is computationally very intensive. Tuning to the optimum frequency found by the GA to design a laboratory experiment might prove to be useful. Furthermore, this approach can be extended to take into account several other laser parameters in addition to its frequency. In multi-dimensional parameter space, the power of the GA will only boost compared to the traditional trial and error search which has been applied to this and similar type of problems.

Sections II and III briefly introduce the physics of the problem. In section III, the short-range potential model is described and some single frequency results in relation to existing published work of Su et al. [4,5] are discussed. In section IV, several pre-processing numerical schemes which can be applied to this problem are presented. This pre-processing step is important because every fitness function evaluation in the genetic algorithm entails the numerical solution of a partial differential equation (PDE). In section V, the GA methodology and its implementation on a parallel machine are discussed. The example simulations are presented in section VI, as well as a comparative study with simulated annealing. In section VII the conclusions are summarized. 


\section{What is Atomic Stabilization?}

The response of atoms and molecules to a well characterized laser field is intriguing and can lead to many interesting applications. For example, one can aim at constructing a "smart" pulse that will drive a chemical reaction down a preferred pathway. Another well known example is harmonic generation, in which an incident photon can be converted into its harmonics. The fundamental equation which governs the dynamics of such a system is the timedependent Schrödinger equation. Numerous computer simulations have been designed in order to study and understand the phenomena involved in the dynamics of an atom exposed to an intense laser field.

In general, to understand the world of atoms and molecules and how they react to an external force, such as a laser field, one needs to construct the Hamiltonian of the system. The Hamiltonian includes the coulomb force which binds the electron to the nucleus, as well as a special term to describe the external force. The Hamiltonian is then plugged into a partial differential equation. In an intense laser field, the external term is big and cannot be treated as a perturbation. Therefore the differential equation, which in this case is the time-dependent Schrödinger equation, becomes nonlinear and interesting nonlinear phenomena can be predicted which are verified experimentally.

Atomic stabilization $[1,2,3,6]$ is one such phenomenon which has been predicted to occur when an atom interacts with high intensity laser fields. The analytical predictions have been followed by numerical simulations $[4,5]$ that are capable of covering regions for which no analytical solutions exist.

To physically understand the underlying picture, one can imagine an electron which is initially bound to the nucleus. Turning the high intensity laser on, the electron (which is described by the wavefunction, the solution to the partialdifferential-equation), is then ejected from the system. This process is known as ionization.

In ionization at low laser intensities, the probability that an electron is stripped from the core atom and becomes ionized linearly increases with intensity. However, the analytical and numerical predictions suggest counterintuitive (nonlinear) dynamics in which at high intensities, the laser works against the ionization process and a new "stabilized" atomic configuration emerges [6]. This surprising effect is known as atomic stabilization. Nowadays, as highintensity laser beams are routinely becoming available, it is possible to test these predictions in a laboratory experiment.

\section{Modeling Atomic Stabilization}

The starting point for any model of atomic stabilization is the time-dependent Schrödinger equation (TDSE),

$$
i \stackrel{\partial \psi}{\partial t}=H \psi
$$

where $\psi$ is the wavefunction, $H$ is the Hamiltonian and $\bar{h}$ is a universal constant. There is no loss of generality if the TDSE is solved in atomic units $(\bar{h}=m=e=1)$.

In a one-dimensional model for the laser-atom interaction, the Hamiltonian $H(x, t)$ contains a nonlinear term to describe the linearly polarized oscillating electric field $E(t)$ along with the kinetic energy (second derivative in space) and the potential energy $V(x)$ :

$$
H(x, t)=-\frac{1}{2} \frac{\partial^{2}}{\partial x^{2}}+V(x)+x E_{0} f(t) \sin (\omega t)
$$

It has been decided to work with an exponentially-decaying short range potential, namely $V(x)=-V_{0} \exp \left(-\frac{x^{2}}{x_{0}^{2}}\right)$. The potential parameters were set at $V_{0}=0.18$ and $x_{0}=7.0225$ so that the potential supports only one bound state, similar to what was done in [7]. In order to compute the initial condition, which is the wavefunction $\psi$ at this bound state, any timedependent expression is taken out of the TDSE and the time-independent Schrödinger equation (TISE) is solved:

$$
\left[-\frac{1}{2} \frac{\partial^{2}}{\partial x^{2}}-V_{0} \exp \left(-\frac{x^{2}}{x_{0}^{2}}\right)\right] \psi=E \psi
$$


This is an eigenvalue problem. The eigenfunction corresponding to the only negative eigenvalue (namely, the bound state) constitutes the initial wavefunction, which is the ground state of the system before the laser is switched on to start the ionization process. For the potential function parameters chosen above, the ground state energy (the negative eigenvalue) comes out to be $-0.0933 \mathrm{au}$.

Substituting (3) into (2), the TDSE with an exponentially decaying short-range potential becomes:

$$
\begin{aligned}
i \frac{\partial \psi(x, t)}{\partial t}= & {\left[-\frac{1}{2} \frac{\partial^{2}}{\partial x^{2}}+x E_{0} f(t) \sin (\omega t)\right.} \\
& \left.-V_{0} \exp \left(-\frac{x^{2}}{x_{0}^{2}}\right)\right] \psi(x, t)
\end{aligned}
$$

This equation will be solved for several laser intensities, i.e. by setting $\left(E_{0}=\sqrt{I_{i}}\right), i=$ $1,2, \ldots$ and using the laser frequency $\omega$ as a parameter to be optimized. That is, the goal of this paper is to come up with the best $\omega$ given a set of intensities $I_{i}, i=1,2, \ldots$ for which the stabilization effect in these intensities is maximal.

Now it is necessary to choose pulse shape $f(t)$ and pulse duration, unless it is decided to vary the shape as a parameter (along with the frequency). As in $[4,5]$, the commonly used procedure is to switch the laser on and off smoothly, according to $f(t)=\sin ^{2}\left((\pi / 2) \times t / T_{1}\right)$ for $0 \leq t \leq T_{1}, f(t)=1$ while the laser is on $\left(T_{1} \leq t \leq T_{2}\right)$ and switching off according to $f(t)=\cos ^{2}\left((\pi / 2) \times t /\left(T_{3}-T_{2}\right)\right)$ for $T_{2} \leq t \leq T_{3}$. In the numerical simulations reported here, $3-10-3$ pulses ( 3 cycles turn-on, 10 cycles fully on, 3 cycles turn-off) were used. The time period of each cycle is $2 \pi / \omega(\omega$ is the laser frequency).

Care should be taken with respect to the boundary conditions. In $[4,5]$ simulations were done specifically for a fixed very-high-frequency value which promoted the ground state well into the continuum. However, in the current studies which include a large range of frequencies, the ground state of the grid walls was found to interfere with the calculations below a certain frequency threshold. This caused a false stabilization peak to appear below this threshold (presumably, a numerical artifact). This prompted the use of either huge grid sizes or any other method such as mask functions, absorbing potentials, or a dilation transformation so that the time-dependent wavefunction will vanish in the limits as $x \rightarrow \pm \infty$ at each time step in the calculation. Mask functions were chosen, taking a $\sin ^{0.2}(x)$ shaped mask function and multiplying with it the wavefunction at the grid edges. In addition, the possibility for a false peak should not be forgotten when working with low frequencies close to the top of the potential well.

Finally, the calculations were done with the following set of numerical parameters in addition to the ones mentioned above. For the spatial grid, $N_{x}=1000$ points are taken ( or $N_{x}=1024$ when Fourier representation is employed). For the mask function region, 100 grid points are used on each side. Grid spacing is $d x=0.1$. For the time grid, $N_{t}=16,000$ steps are used corresponding to a 3-10-3 pulse (total of $16 \mathrm{cy}-$ cles) in which each cycle contains 1000 time steps. Time spacing is determined by the laser frequency, so that $d t=T / n d t$ where $T$ is the time-period of a cycle obtained from the frequency and $n d t=1000$ time steps per cycle. Unless noted otherwise, atomic units are used for all these parameters.

Figure 1 shows the end-of-pulse ionization vs. intensity plots (stabilization curves) for two different laser frequencies (or, wavelengths). The curves were obtained by solving the TDSE for $\psi_{f}\left(x, T_{d}\right)$, where $T_{d}$ is the duration of the pulse. After the pulse duration is over, $\psi_{f}$ is projected onto $\psi_{i}$ so that $p 0=\left|\left\langle\psi_{f} \mid \psi_{i}\right\rangle\right|^{2}$ is the probability of the final wavefunction being in the ground state. $1-p 0$ is plotted as a function of intensity, after solving the TDSE repeatedly for different intensities, as a measure of the ionization rate. Full ionization is achieved at 1.0. For low intensities, one can see the expected linear response, which is also predicted using perturbation theory. For high intensities, however, a stabilization structure can be seen whereby the laser interferes with the ionization process and the ionization is suppressed. Perturbation theory breaks down and fails to explain the behavior at such high intensities.

In the next few sections, the model which accounts for the stabilization structure will be formulated as an optimization problem. Both a 


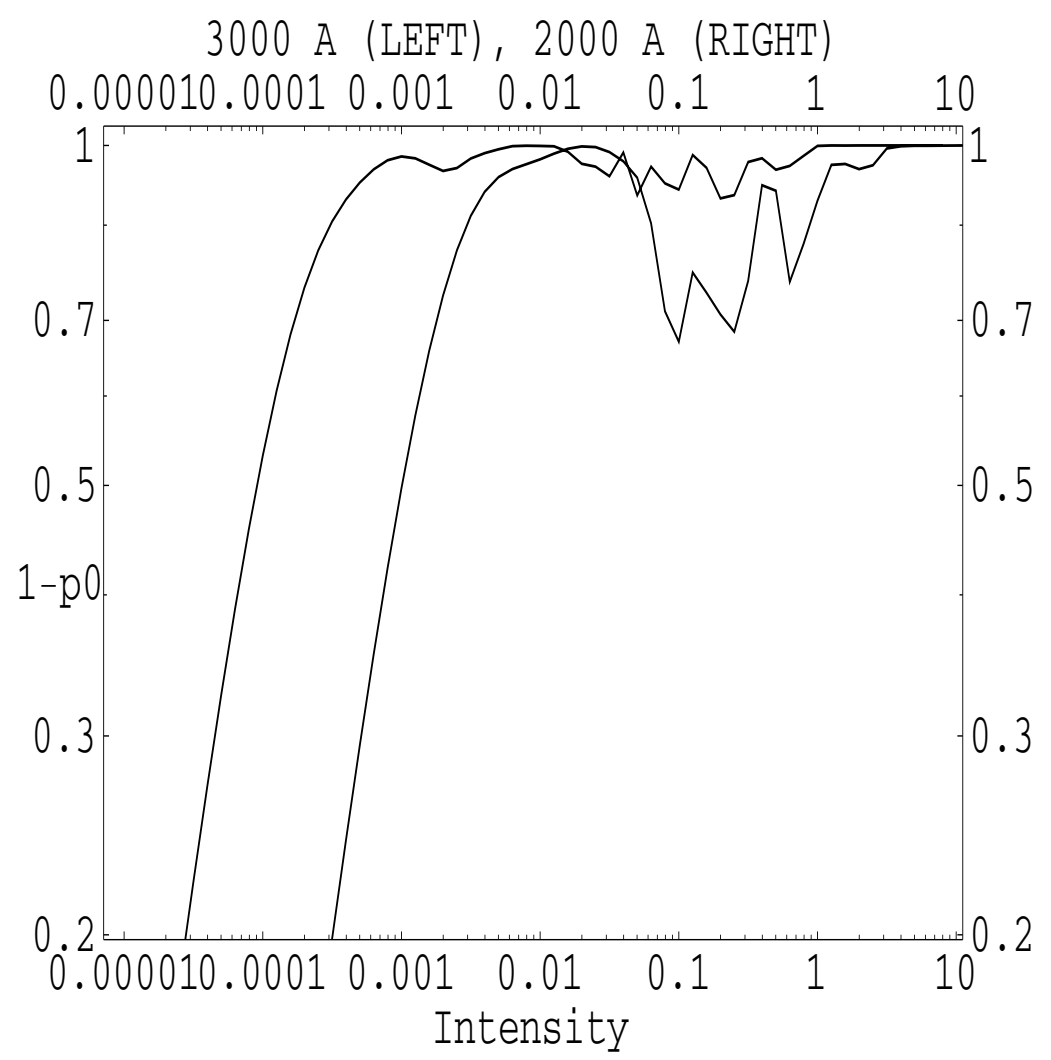

Fig. 1. End of pulse ionization 1-p0 as a function of laser intensity, for two different frequencies. The 16-cycle pulse that was used consists of a 3-cycle smooth (sine-squared ramp) turn-on, a 3-cycle smooth turn-off and 10-cycle constant laser field inbetween. Ionization suppression at high intensities is evident in both cases. Note that for the left curve, which corresponds to $\omega=0.152(\lambda=3000 \AA)$, the grid walls start interfering in the calculation producing a slight minimum in the structure around $I=0.001$. This frequency is a 1-photon promotion from the ground state to slightly above the top of the well. The minimum will sharpen as the frequency is lowered. For higher frequencies, no minimum appears but the curve is shifting to the right, towards the higher intensity region. The right curve corresponds to a frequency of $\omega=0.228(\lambda=2000 \AA)$. In atomic units, $I=1.0$ corresponds to

$$
I=3.5 \times 10^{16} \mathrm{~W} / \mathrm{cm}^{2} \text {. }
$$

genetic algorithm and simulated annealing approach will be implemented to search for the best parameters to achieve this nonlinear effect. Such a simulation will prove to be highly useful and relevant to the physics behind this phenomenon and will lead to a new finding regarding the role of laser frequencies in atomic stabilization $[8]$.

\section{Computational Challenge in Fitness Function Evaluation}

Solving the time-dependent Schrödinger equation (TDSE) using grid methods is central to the fitness function evaluation. Therefore, choosing the most efficient scheme to integrate the TDSE is of high importance in the simulation.

In an atomic stabilization problem, as well as works on ATI (above threshold ionization) and time-dependent multiphoton process studies [9], the Crank-Nicolson $(\mathrm{CN})$ method was used with a computational effort of $O(N)$ [10] since the LU decomposition to invert the resulting tridiagonal matrix only costs $N$ operations. Therefore, even the second order difference scheme SOD [11] (an explicit version of the implicit CN method) will not result in less operations for a one-dimensional problem. All other methods which rely on the FFT $[12,13,14,15,16]$, cost at least $N \log (N)$ operations. Therefore, unless one of these methods offers either an efficient representation of the derivative operator with less grid points $N$ or an efficient propagator to be used, the $\mathrm{CN}$ method should clearly be the method of choice.

It is instructive to point out that in several cases, apart from stabilization, some of these methods are indeed more efficient than the $\mathrm{CN}$ method. 
With the harmonic oscillator potential, for example, $N$ can be reduced by a factor of 5 due to an efficient spatial representation $[13,16]$. With a gaussian shaped pulse, a Fourier representation of this gaussian in time can lead to a Chebychev expansion of the time evolution operator [14] which is known to converge extremely fast in time-independent problems. The Hamiltonian in stabilization, however, consists of a short-range potential and a pulse shape $f(t)$ which is much harder to represent efficiently with a Fourier expansion without additional cost. A separation of the fast oscillations from the smooth pulse [15] is an interesting approach which can be tried. Nevertheless, it is clearly not obvious whether "smart" time propagation schemes such as the $\left(t, t^{\prime}\right)$ or the variable timestepping scheme [17] can reduce the amount of CPU time calculations in stabilization problems.

Attention is therefore turned to the spatial representation, comparing the $\mathrm{CN}$ method with the well known Split Operator Method (SPO) [12]. Specifically for stabilization problems, SPO has been widely used $[4,5,8]$. With the SPO, time propagation is performed by approximating the propagator,

$$
e^{-i H \Delta t}=e^{-i K \Delta t / 2} e^{-i V \Delta t} e^{-i K \Delta t / 2}+O\left(\Delta t^{3}\right)
$$

where $H=V+K, H$ is split into the potential term $V$ and the kinetic term $K$ which includes all spatial derivatives. The spatial derivatives will then be numerically calculated by performing an FFT on the wavefunction $\psi$, multiplying the result by a diagonal matrix, then transforming back to configuration space by an inverse FFT. The method makes use of a Fourier representation whereas in the $\mathrm{CN}$ method, the quantity

$$
e^{-i H \Delta t}=(1-i H \Delta t / 2)(1+i H \Delta t / 2)^{-1}+O\left(\Delta t^{3}\right)
$$

in which all spatial derivatives in $H$ are numerically calculated, uses a finite-difference representation. As both methods are second order in time, the question arises as to which representation costs less CPU time. Since $\mathrm{CN}$ costs $\mathrm{O}(\mathrm{N})$ and the FFT costs $N \log (N)$, the hope is that the Fourier representation will permit a smaller number of grid points $N$. However, since the time-dependent wavefunction $\psi$ is not a nicely behaving function when driven by a high intensity laser field, it was found that the derivative operator on a grid needs to be represented with the same grid parameters $N_{x}, \Delta x$ in both methods in order to get the same numerical results in practice. Therefore, the cost in CPU time is simply based on the number of operations per time step required in each method. On the CRAY T3E, in which a built-in enhanced FFT routine is used, it is at least five times faster in favor of the CN over SPO.

To ensure that no loss of accuracy is suffered, the CN method was compared with the SPO method, both practically yielding the same output. Therefore, $\mathrm{CN}$ was chosen as the method of choice for generating each point in the endof-pulse probability vs. intensity curve (stabilization curve, figure 1).

In the next section, a fitness function to analyze stabilization curves is formulated. The goal is to identify, given such a curve, where the stabilization structure is most pronounced.

\section{Genetic Algorithms on a Parallel Platform}

The goal is to maximize:

$$
\begin{aligned}
f(\omega)=- & N \times p 0_{I_{i}}+p 0_{I_{i+1}}+p 0_{I_{i+2}}+\ldots+p 0_{I_{i+N / 2}} \\
& +p 0_{I_{i-1}}+p 0_{I_{i-2}}+\ldots+p 0_{I_{i-N / 2}}(8)
\end{aligned}
$$

given $p 0_{I_{i}}\left(I_{i}\right.$ is the intensity and $p 0$ is the projection to the ground state) and the projections to the ground state of the neighboring intensities, namely $p 0_{I_{i+1}}, p 0_{I_{i+2}}, \ldots, p 0_{I_{i-1}}, p 0_{I_{i-2}}$, $\ldots$, where $N$ is the number of neighboring intensities. Equation (8) is equivalent to equation (1). This quantity is greatest for the most pronounced minimum in the curve, centered at $I=I_{i}$. It is frequency-dependent and maximizing this function guarantees to locate the optimal frequency $\omega$ for which the minimum caused by stabilization is most pronounced. Possible ways to perform the search for optimal frequencies are trial-and-error, enumerative and random search techniques.

There are several reasons why a genetic algorithm $[18,19]$ was chosen for the implementation. First, it is impossible to know a priori how 
many minima and maxima are hidden in the fitness function. By trial and error one can sense that there are optimal frequencies, but there is a likelihood to miss the best frequency in an unexpected place, since there might be several resonant frequencies. Second, it is laborious and a waste of resources to perform a trial-anderror search when the evaluation of the fitness function is costly. An automated search would be beneficial. Third, an extension to several other parameters is straightforward.

Since the evaluation of the fitness function is costly, a variant of genetic algorithms which can offer a saving in the number of function evaluations is most desirable, particularly for the simulation in this paper. Micro genetic algorithms $(\mu G A)[20]$ is an approach which was shown to reach a near-optimal region faster than simple genetic algorithms (SGA) on several test functions. As the name 'micro' suggests, $\mu G A$ works with a small population size (typically, 5) and aims at finding the optimum as quickly as possible through a best-so-far string without improving any average performance. Therefore, $\mu G A$ was implemented in addition to the SGA and simulated annealing for the comparative study done in the next section.

In evaluating the fitness function, it is possible to perform the calculation for several intensities all at once. A parallel platform is most suited for this task, since different intensities can be distributed among several processors that perform the same calculation, each processor working on a different point or set of points in a stabilization curve.

MPI (Message Passing Interface) can be used to communicate between the different processors and is currently available on almost all parallel platforms. Since the calculation for each intensity is done independently and only at the very end the fitness function is calculated based on information from all processors, it is desirable to reduce the amount of communication. In this problem, it is possible to refrain completely from any need of communication, assuming that enough processors are available to handle each point in the minimum and surrounding intensities. Therefore, each intensity is assigned to a single processor and a synchronizing barrier makes sure that all processors are done calculating their end-of-pulse probabilities before proceeding onwards. Only then the master proces- sor (proc. 0) collects the results from a file and calculates the fitness function.

The various steps in the simulation are as follows:

1. Generate an initial population of frequencies $\omega_{1}^{(0)}, \omega_{2}^{(0)}, \omega_{3}^{(0)}, \ldots$

2. For each $\omega_{j}^{(0)}$ in the population, solve the TDSE in parallel on $(\mathrm{N}+1)$ processors for $(\mathrm{N}+1)$ given intensities, $I_{i}, I_{i+1}, I_{i+2}, \ldots, I_{i-1}, I_{i-2}, \ldots$, to obtain end-of-pulse ionization probabilities $p 0_{I_{i}}, p 0_{I_{i+1}}, p 0_{I_{i+2}}, \ldots, p 0_{I_{i-1}}$, $p 0_{I_{i-2}}, \ldots$ and evaluate the fitness function $f_{j}^{(0)}=-N \times p 0_{I_{i}}+p 0_{I_{i+1}}+$ $p 0_{I_{i+2}}+\ldots+p 0_{I_{i-1}}+p 0_{I_{i-2}}+\ldots$ serially on the master processor.

3. Build next generation $\omega_{j}^{(1)}$ (in general $\omega_{j}^{(k)}$ for the kth generation) using genetic operators and evaluate new fitness function $f_{j}^{(1)}$.

4. Stop when $f_{\text {best-so-far }}$ does not improve substantially after several generations. Print $\omega^{*}$ corresponding to $f^{*}$. Done.

\section{Go to step 3.}

To summarize, in order to maximize the fitness function, $\mathrm{p} 0$ is calculated for each intensity. This operation amounts to solving a single PDE. For a 16-cycles pulse, this can be achieved in approximately 150 seconds on an Ultra-Sparc. Working on the UC Berkeley NOW (Network of Workstations), which is a collection of UltraSparcs hooked together, it is possible to calculate all p0's at once so that each evaluation function costs around 150 seconds. For the genetic implementation, a genetic algorithm driver written in FORTRAN was used $[21,22]$. While a number of processors are used to calculate the fitness function, the master processor by itself is used to advance the simulation from one generation to the next until convergence is achieved. 


\section{Experiments: Genetic Algorithms vs. Simulated Annealing}

To begin with, SGA was tested for two cases to identify the optimal frequency to achieve maximum stabilization. In the first case, the goal was to find maximum stabilization around $I=0.1$ au $\left(I=3.5 \times 10^{15} \mathrm{~W} / \mathrm{cm}^{2}\right)$. Surrounding intensities were taken to be at $I=$ $1.5 \times 10^{15} \mathrm{~W} / \mathrm{cm}^{2}, I=2.5 \times 10^{15} \mathrm{~W} / \mathrm{cm}^{2}, I=$ $4.5 \times 10^{15} \mathrm{~W} / \mathrm{cm}^{2}, I=5.5 \times 10^{15} \mathrm{~W} / \mathrm{cm}^{2}$. In the second case, the optimal frequency for finding maximum stabilization around $I=0.2$ au was calculated by the GA. The frequency region is extended between $\lambda=1500 \AA$ to $\lambda=3500 \AA$, since above that would promote the ground state to below the top of the potential well and below that the stabilization effect disappears. However, one of the virtues of a GA automatic search is that if such prior knowledge is obscured, it is still possible to pick a larger region without covering more space by a tedious trial-and-error search. An additional calculation confirmed that taking a frequency region between $\lambda=0$ to $\lambda=5000 \AA$ converges to the same optimum but takes more CPU time. The results in figure 3 were obtained after 250 function evaluations of the GA run, when strict convergence was achieved. This was done with a population size of $N=50$, stopping the calculation after 5 generations. GA parameters were set to: crossover percentage of 0.5 (uniform crossover is used), mutation percentage of 0.02 , tournament selection and elitism was used (best individual replicated into next generation). The frequency region between $\lambda=0$ to $\lambda=3500 \AA$ was divided into 1024 equal segments. In both plots of figure 3 , the reported optimal frequency was obtained after repeating the computational experiment for ten times.

Several observations are important before initiating a comparative study between different stochastic search strategies. First, with only 50 function evaluations, near optimality was achieved in all repeated runs. That is, after 10 consecutive runs of the GA for the second test-case, the average of the best-so-far-fitness was 0.544 corresponding to the frequency of $\lambda=2223.4^{\circ} \mathrm{A}$ while the lowest and highest values of the best-so-far-fitness corresponded to the frequencies $\lambda=2209.7^{\circ}$ and $\lambda=2242.9{ }^{\circ}$, respectively. This difference is of no importance from the experimentalist point of view. Therefore, strict optimality is not always necessary and stopping the calculation after only 50 function evaluations is satisfactory. Second, each function evaluation takes several hours on the parallel platform which was used. A physicist cannot afford to spend several days performing repeated runs in the laboratory; instead, the aim will be to use as few function evaluations as possible along with a scheme which is reliable enough to meet its purpose. However, for the comparative analysis conducted in this paper, numerous repeated runs with the GA were performed and 664 distinct function evaluations were gathered. This enabled to plot the fitness function (figure 2) and continue the handful of runs needed for the statistical analysis using a fourth-order interpolating polynomial, after it was checked that identical results are achieved using the parallel platform and the interpolating function for performing fast function evaluations. It is now possible to check whether $\mu \mathrm{GA}$ or simulated annealing can offer a reduction in computational cost compared to the SGA which is used.

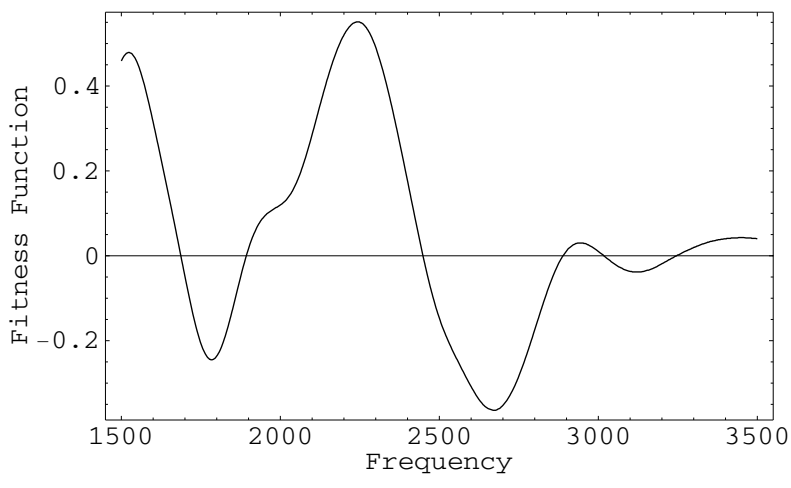

Fig. 2. A plot of the fitness function, which was not known a priori, based on 664 function evaluations gathered in the course of the GA implementation. It is seen that the function is multi-modal in the desired frequency range, and can be considered continuous to a good approximation. Therefore an interpolating function can be used in further function evaluations which are necessary for a comparative analysis.

In figure $4, \mu \mathrm{GA}$ performance is compared with both SGA and SA (Simulated Annealing) for the second case shown in figure 3 . The number of function evaluations is taken as a measure of computational effort and the best-so-far fitness is the measure of performance. Average performance at each generation is of no interest in $\mu \mathrm{GAs}$, therefore the best-so-far criterion is the one which fits the comparison. Population size 


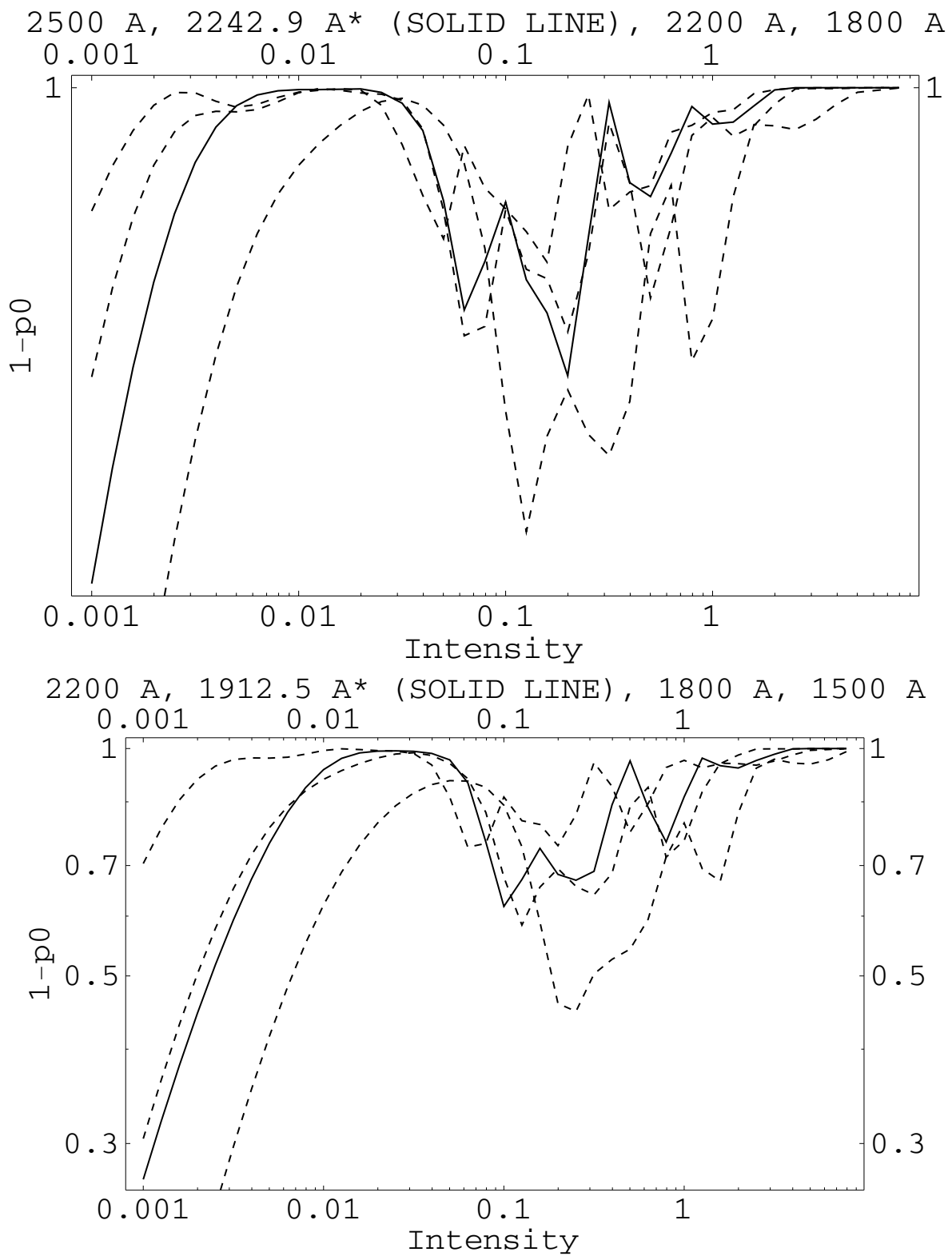

Fig. 3. End of pulse ionization as a function of laser intensity, for several distinct frequencies. Same parameter values as in figure 1 apply. In the upper plot, $\lambda=1912.5^{\circ} \mathrm{A}$ corresponds to the best frequency the genetic algorithm found so that a stabilization structure surrounding $I=0.1$ can be observed. In the lower plot, $\lambda=2242.9 \AA$ corresponds to the best frequency the GA found for a stabilization structure surrounding $I=0.2$.

for the $\mu \mathrm{GA}$ was chosen to be 5 along with a crossover percentage of 0.5 . In $\mu \mathrm{GA}$, no mutation is performed since a random shuffle guards from fixation. For the SA approach, a package available at [23] was used which is based on [24]. The simulated annealing driver was attached to the stabilization problem of this paper with initial values recommended for the test function in [24]. Then, the parameters were further tuned to best locate the optimal frequency which was achieved by the GA, starting from an initial guess of $\lambda=1500 \AA$. SA parameters were set to: initial temperature of 5.0, with a decline in temperature $T$ according to $T(i+1)=R T \times T(i), R T=0.85$, where $i$ is the ith iteration (each iteration containing 5 function evaluations with the current temperature). With a simulated annealing, unlike GAs, one starts from a single initial guess within the frequency range. Therefore, 50 runs were performed after dividing the region into 50 equally spaced points so that in the end, all parts of the 

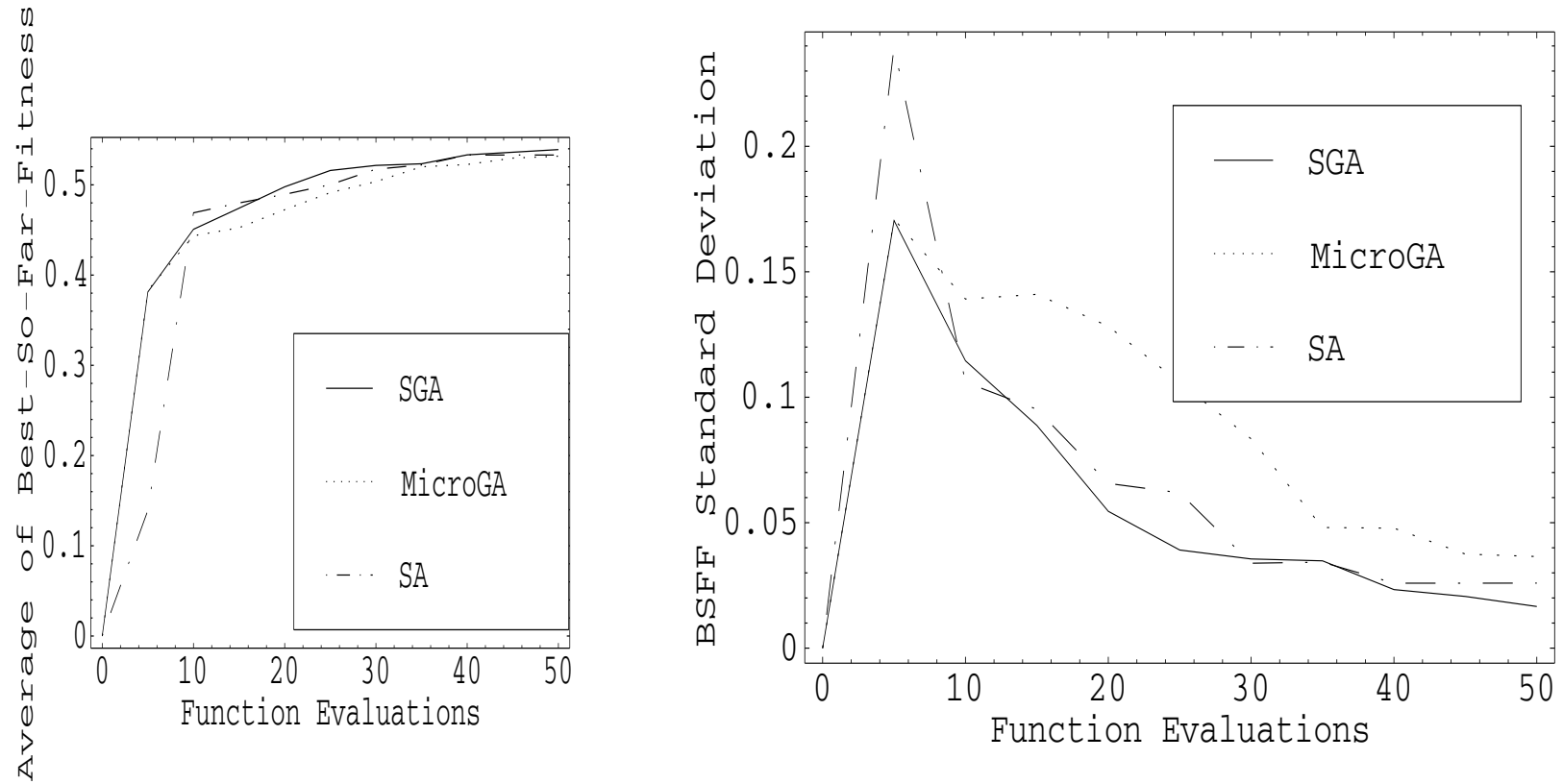

Fig. 4. Performance comparison of SGA (solid line), SA (dashed-dotted line) and $\mu \mathrm{GA}$ (dotted line) to find the optimal frequency for the bottom case in figure 3. The average and standard deviation of 50 consecutive runs in each method was taken, and performance of the 3 methods based on these measures show they are almost equally successful in finding the optimum. For the particular function to be optimized in this paper (figure 2 ), $\mu \mathrm{GA}$ does not seem to result in a reduction of computational cost.

region will be taken into account.

Examining the average and standard deviation of the 3 methods (figure 4), it is seen that they are all reaching the same optimum value at about the same speed and the differences are small. There is no gain in applying $\mu \mathrm{GA}$ for this particular function, although it might be beneficial to implement this scheme along with SGA for other functions. SA also performs well for this particular function. SGA is most reliable (least standard deviation) and can be safely used to search for the optimal frequency in a systematic way.

\section{Conclusion}

Using a parallel platform to solve the timedependent Schrödinger equation for several intensities at once, a GA was used to predict the optimal laser frequency to achieve atomic stabilization. The difficult issues needed to be addressed in such a simulation are which PDE solver is most efficient, how to distribute the workload on several processors and whether a GA approach can be found which reduces the number of expensive function evaluations to locate the optimum.
It should be noted that the paper does not aim to contribute any new ideas in GA research, nor has it designed a new GA method for solving a particular problem. The paper has applied an existing GA method to an important problem in experimental physics. For this particular case, it appears that all three methods are competitive, with no particular method claiming any advantage.

Using the Berkeley Network of Workstations, optimal frequencies for two intensity regions were successfully identified. Future work might extend the model from one dimension to two dimensions, or include several laser parameters apart from the frequency, such as the pulse shape and duration, which are best to be used in a laboratory experiment. The GA was found to be a reliable search technique for future atomic stabilization and related models in which the timedependent Schrödinger equation is needed to be solved numerous times.

\section{Acknowledgments}

The authors would like to acknowledge helpful discussions with K.C. Kulander. One of the authors (DB) thanks W. Hugh Woodin for the 
fine hospitality at the UC Berkeley Mathematics department. The genetic algorithm front-end driver code, written by D.L. Carroll, was used in this work. Computing resources were supported by NPACI (National Partnership for Advanced Computational Infrastructure). Work was performed on the SDSC (San-Diego Super Computer Center) Cray T3E and the UC Berkeley NOW (Network Of Workstations). AEO and DB acknowledge support provided by the National Science Foundation under grant PHY-9322067.

\section{References}

[1] Geltman S. and Teague J., (1974) J.Phys.B 7, L22.

[2] Gersten J.I. AND Mittleman M.H., (1976), J.Phys.B 9, p.2561.

[3] Gavrila M. AND KaminsKi J.Z., (1984), Physical Review Letters 52, 614.

[4] Su Q., IRVING B.P., EberLy J.H., (1997) "Ionization Modulation in Dynamic Stabilization", Laser Physics, Vol.7, No.3, p.568.

[5] Su Q., IRving B.P., JohnSOn C.W., EBERLY J.H., (1996) "Stabilization of a one-dimensional shortrange model atom in intense laser fields", J.Phys.B: At.Mol.Opt.Phys.29, p.5755.

[6] EBerly J.H. AND Kulander K.C., (1993) "Atomic Stabilization by Super-Intense Lasers", Science, Vol 262, p.1233.

[7] Bardsley J.N., SZOKe A., COMElla M.J., (1988) "Multiphoton ionisation from a short-range potential by short-pulse lasers", J.Phys.B., Vol.21, p.3899.

[8] BARASH D., OREL A.E., BAER R., (2000) "Laser Induced Resonance States as a Cause for Dynamic Suppression of Ionization in High Frequency Short Pulses", Physical Review A, Vol. 60, 013402.

[9] Kulander K.C., Schafer K.J., Krause J.L., (1992) "Time-Dependent Studies of Multiphoton Processes", in Gavrila M.,Ed.; Atoms in Intense Radiation Fields, Academics, New-York, p.247300.

[10] Press W.H., TeuKolsky S.A., VetTerling W.T., FLANNERY B.P., (1992) Numerical Recipes, Second Edition, Cambridge University Press.

[11] ASKAR A., CAKMAK A.S., (1978) "Explicit Integration Method for the Time-Dependent Schrödinger Equation for Collision Problems", J.Chem.Phys., 68(6), p.2794.

[12] FeIT M.D.,FleCK J.A.,STEIGER A., (1982) “Solution of a Schrödinger Equation by a Spectral Method", J.Comp.Phys.47, p.412-433.
[13] Kosloff R., KoslofF D., (1983) “A Fourier Method Solution for the Time-Dependent Schrödinger Equation", J.Chem.Phys., Vol.79, No.4, p.1823.

[14] Peskin U., Kosloff R., Moiseyev N., (1994) “The Solution of the Time-Dependent Schrödinger Equation by the $\left(t, t^{\prime}\right)$ Method: The Use of Global Polynomial Propagators for Time-Dependent Hamiltonians", J.Chem.Phys.100(12), p.8849-8855.

[15] YAO G., WyATT R.E., (1994) "Stationary Approaches for Solving the Schrödinger Equation with Time-Dependent Hamiltonians", J.Chem.Phys.101 (3), p.1904.

[16] Kosloff R., (1996) “Quantum Molecular Dynamics on Grids", in Wyatt R.E., Zhang J.,Ed.; Dynamics of Molecules and Chemical Reactions, Marcel Dekker, New York.

[17] CERJAN C., Kosloff R., (1993) "Efficient Variable Time-Stepping Schemes for Intense Field-Atom Interactions", Physical Review A., Vol.47, No.3, p.1852.

[18] Holland J.H., (1975) “Adaptation in Natural and Artificial Systems", MIT Press, Cambridge, MA.

[19] GoldBerg D.E., (1989) "Genetic Algorithms in Search, Optimization and Machine Learning", Addison-Wesley, Reading, MA.

[20] KRISHNAKUMAR K., (1989) "Micro-Genetic Algorithms for Stationary and Non-Stationary Function Optimization", SPIE: Intelligent Control and Adaptive Systems, Vol. 1196, Philadelphia, PA.

[21] CARROLL D.L., (1996) “Chemical Laser Modeling with Genetic Algorithms", AIAA J., Vol.34, 2, p.338-346.

[22] CARroll D.L., (1996) "Genetic Algorithms and Optimizing Chemical Oxygen-Iodine Lasers", in Wilson H.B. et al., Eds.; Developments in Theoretical and Applied Mechanics, Vol. XVIII, The Univeristy of Alabama, p.411-424.

[23] http://emlab.berkeley.edu/Software/abstracts/ goffe 895.html

[24] GOFFe, FERrIER AND RoGers, (1994) “Global Optimization of Statistical Functions with Simulated Annealing", Journal of Econometrics, Vol.60, no.1/2, p.65-100.

Received: June, 1998 Revised: May, 1999 Accepted: January 2000

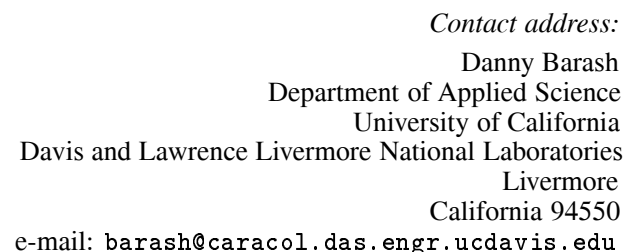

Contact address:

Danny Barash Univer of Applied Science aboratories California 94550 e-mail: barash@caracol.das.engr.ucdavis.edu 
DR. DANNY BARASH received the B.Sc. degree in physics from the Hebrew University, Jerusalem, Israel, in 1992, and the M.Sc. and Ph.D. degrees in applied science from the University of California, Davis, in 1996 and 1999, respectively. He is currently a research and development engineer at Hewlett-Packard Laboratories Israel. His research interests include computational physics, scientific computing and image processing.

DR. ANN E. OREL received the Ph.D. degree in chemistry from the University of California, Berkeley, in 1981. She is currently a professor in the Department of Applied Science, University of California, Davis, and the Lawrence Livermore National Laboratories. Her research interests include theoretical atomic and molecular physics.

DR. V. RAO VEMURI, a professor in the Department of Applied Science, University of California, Davis, also holds joint appointments with the Department of Computer Science and with the Lawrence Livermore National Laboratory. His teaching and research interests are in artificial neural nets, genetic algorithms, web-based applications and modeling and simulation. He served as the Editor-in-Chief of the CS Press of IEEE during 1990-92. He is the founding president of Eco Foundation, a non-profit organization dedicated to promote public participation in matters of ecology and environment. 